\title{
Squared interaction matrix Sherrington-Kirkpatrick model for a spin glass
}

\author{
D. S. Dean, ${ }^{1, *}$ and F. Ritort ${ }^{2, \dagger}$ \\ ${ }^{1}$ IRSAMC, Laboratoire de Physique Quantique, Université Paul Sabatier, 118 route de Narbonne, 31062 Toulouse Cedex 04, France \\ ${ }^{2}$ Departament de Física Fonamental, Facultat de Física, Universitat de Barcelona Diagonal 647, 08028 Barcelona, Spain
}

(Received 19 December 2001; revised manuscript received 12 March 2002; published 10 June 2002)

\begin{abstract}
The mean-field theory of a spin glass with a specific form of nearest- and next-nearest-neighbor interactions is investigated. Depending on the sign of the interaction matrix chosen we find either the continuous replica symmetry breaking seen in the Sherrington-Kirkpartick model or a one-step solution similar to that found in structural glasses. Our results are confirmed by numerical simulations and the link between the type of spin-glass behavior and the density of eigenvalues of the interaction matrix is discussed.
\end{abstract}

DOI: 10.1103/PhysRevB.65.224209

PACS number(s): 75.10.Nr, 75.30.Fv, 75.50.Lk

\section{INTRODUCTION}

The Sherrington-Kirkpatrick (SK) model $^{1}$ is the most well-known example of a disordered and frustrated system in the field of spin glasses. ${ }^{2}$ It corresponds to the infinite-range version of the Edwards-Anderson (EA) model introduced earlier in $1975 .{ }^{3}$ In the EA model quenched disorder is introduced in the random sign of the exchange couplings between nearest-neighbor spins on a lattice. The infinite-ranged version is the natural mean-field version of the EA model, in the same sense as the infinite-range ferromagnet is the meanfield theory for the Ising model. A nearly complete solution of the SK model has been found ${ }^{4}$ which has raised subtle questions about the nature of the spin-glass phase showing that the mean-field theory of spin glasses is considerably more complex than the standard mean field of, say, ferromagnetic systems. Plenty of questions were posed after it was shown that the correct thermodynamic solution had to be understood in terms of a replica-symmetric broken solution. For instance, does the ergodicity breaking (implied by replica symmetry breaking) also occur in short-range systems? Also, has replica symmetry a true physical meaning inasmuch as time-reversal symmetry has for usual ferromagnets? After many years of research these questions have turned out to be extremely difficult and in the meantime criticisms questioning their relevance regarding our understanding of experimental systems have also been raised. Scaling theories of finite-dimensional spin glasses, so-called droplet models, ${ }^{5}$ seem to be at variance with the image of replica symmetry breaking. It is thus useful to introduce new solvable models which correspond to mean-field versions of different finitedimensional problems in order to improve our understanding of the spin-glass problem.

In this paper we introduce a solvable spin-glass model which corresponds to the mean-field version of the EdwardsAnderson model but includes next-nearest-neighbor interactions in a particular way. We will refer to this model as the squared interaction matrix (SIM) SK model. The motivation is that the model admits the possibility of being realized on a finite-dimensional lattice and incorporates correlations between the first-nearest-neighbor and second-nearest-neighbor coupling interactions. At a first glance it seems strange to consider a next-nearest-neighbor interaction in a totally connected spin-glass model; however, we shall see that the cor- relations introduced in the couplings can lead to a physics different to that of the SK model. In addition this model allows one to discuss the role of the density eigenvalues of the interaction matrix in the spin-glass behavior.

\section{MODEL}

The model we study is a totally connected one with Hamiltonian

$$
H=-\frac{1}{2} \sum_{i j} K_{i j} S_{i} S_{j},
$$

the spins $S_{i}(1 \leqslant i \leqslant N)$ being Ising spins taking the values \pm 1 . Here we take the interaction matrix $K$ to be of a squared interaction type:

$$
K=J J^{T},
$$

where the $J_{i j}$ are independent Gaussian random variables such that $\bar{J}_{i j}=0$ and $\overline{J_{i j} J_{k l}}=\delta_{i k} \delta_{j l} / N$. Here the overbar denotes the disorder averaging and we note that the matrix $J$ here is not symmetric. The same model with $J$ symmetric may be studied; however, taking $J$ as nonsymmetric considerably simplifies the analytical study of the model. We note that, in finite dimensions, if $J$ were a next-nearest-neighbor interaction, then $K$ is a matrix which has interactions between next nearest neighbors.

In this paper we will consider two models: the positive temperature model where $K=J J^{T}$ (we will refer to this as the $K>0$ model) and the negative temperature model where $K$ $=-J J^{T}$ (we will refer to this as the $K<0$ model). Contrary to what happens in the usual Sherrington-Kirkpatrick model, the model (1) is not invariant under the transformation $K \rightarrow$ $-K$. Clearly $K$ is a matrix which is positive definite and $-K$ a matrix which is negative definite.

For a symmetric matrix $J$ taken from the Gaussian ensemble, the density of eigenvalues $\lambda$ is given by the Wigner semicircle law ${ }^{6}$

$$
\rho_{J}(\lambda)=\frac{1}{2 \pi} \sqrt{4-\lambda^{2}},
$$

with $\lambda \in[-2,2]$. In the corresponding spherical spin model, ${ }^{7}$ at low temperature, the system minimizes its energy via a 
macroscopic condensation onto the eigenvector corresponding to $\lambda=2$. In the case of the SK model this condensation is not possible due to the discrete nature of the spins; however, it seems reasonable to assume that the density $\rho(\lambda)$ for $\lambda$ $\sim 2$ plays an important role in the low-temperature behavior. In our model, if $J$ is taken to be symmetric, the density of eigenvalues $\kappa$ is clearly given by

$$
\rho_{ \pm K}(\kappa)=\frac{1}{4 \pi \sqrt{ \pm \kappa}} \sqrt{4 \mp \kappa}
$$

where now $\kappa \in[0,4]$ in the $+K$ or $K>0$ model and $\kappa \in$ $[-4,0]$ for the $-K$ or $K<0$ model. In fact one can show that ${ }^{9}$ this is also the density of eigenvalues in the case of $J$ nonsymmetric. Hence, in the positive case, the low-energy region of the interaction matrix has the same form as the SK model as one would have in a spherical model a condensation onto the eigenvector of the largest eigenvalue. In the negative case, however, the largest eigenvalues are at $\kappa=0$, but the density of eigenvalues now diverges in this region. In the corresponding spherical model it is easy to see that this eliminates the finite-temperature phase transition. This happens in exactly the same way that the divergence of the density of occupation of the zero-energy states for free bosons in two or less dimensions eliminates the finite-temperature Bose-Einstein transition.

In the following treatment we consider the $K>0$ model, although the same mathematical treatment we present here can be applied to the $K<0$ case on changing the sign of the inverse temperature $\beta$. The Hamiltonian of the model can thus be written as

$$
H=-\frac{1}{2} \sum_{i j k} J_{i k} J_{j k} S_{i} S_{j},
$$

and the partition function for the model is therefore given by

$$
Z=\operatorname{Tr}_{S_{i}} \exp \left[\frac{\beta}{2} \sum_{i j k} J_{i k} J_{j k} S_{i} S_{j}\right] .
$$

In order to facilitate taking the disorder average we make a Hubbard-Stratonovich transformation by introducing the auxiliary Gaussian spins $x_{i}$ to obtain

$$
Z=\operatorname{Tr}_{S_{i}, x_{i}} \exp \left[\beta \sum_{i j} J_{i j} S_{i} x_{j}\right],
$$

where $\operatorname{Tr}_{x}$ indicates the trace over the Gaussian spin $x$ and is defined by

$$
\operatorname{Tr}_{x}=\int d x \sqrt{\frac{\beta}{2 \pi}} \exp \left(-\frac{\beta x^{2}}{2}\right) .
$$

In the form (7) the model is that of an asymmetric Little model ${ }^{8}$ but where one of the two sets of spins is Gaussian rather than Ising. Interest in the Little model arose one decade ago, in the context of neural networks as the parallel dynamics of the standard Hopfield model ${ }^{10}$ coincides with the sequential dynamics in the Little model. ${ }^{11}$ The corresponding mean-field spin-glass model was studied in Ref. 12 where it was shown that the equilibrium behavior is the same for both the SK and Little (for both asymmetric and symmetric couplings) models. Here we have verified numerically that, in our model also, a symmetric or asymmetric matrix leads to the same physical behavior.

\section{POSITIVE-TEMPERATURE $K>0$ MODEL}

We introduce replicas of both the Gaussian and Ising spins in order to average over the disorder via the replica method, obtaining

$$
\begin{aligned}
\overline{Z^{n}} & =\operatorname{Tr}_{S_{i}^{a}, x_{i}^{a}} \exp \left[\frac{\beta^{2}}{2 N} \sum_{i j} \sum_{a b} S_{i}^{a} x_{j}^{a} S_{i}^{b} x_{j}^{b}\right] \\
& =\operatorname{Tr}_{S_{i}^{a}, x_{i}^{a}} \exp \left[\frac{\beta^{2} N}{2} \sum_{a b} Q_{a b} P_{a b}\right]
\end{aligned}
$$

where we have introduced the Ising and parabolic spin overlaps

$$
Q_{a b}=\frac{1}{N} \sum_{i} S_{i}^{a} S_{i}^{b} \quad \text { and } \quad P_{a b}=\frac{1}{N} \sum_{i} x_{i}^{a} x_{i}^{b} .
$$

The trace over the Ising spins is accomplished using a $\delta$-function representation of the overlap constraint:

$$
\begin{aligned}
\operatorname{Tr}_{S_{i}^{a}} \delta\left(\frac{N}{2} Q_{a b}-\sum_{i} S_{i}^{a} S_{i}^{b}\right) \\
=\frac{1}{(2 \pi)^{n^{2}}} \operatorname{Tr}_{S_{i}^{a}} \int d \Lambda_{a b} \\
\\
\quad \times \exp \left(\frac{N}{2} \sum_{a b} \Lambda_{a b} Q_{a b}-\frac{1}{2} \Lambda_{a b} \sum_{i} S_{i}^{a} S_{i}^{b}\right) \\
=\frac{1}{(2 \pi)^{n^{2}}} \int d \Lambda_{a b} \exp \left(\frac{N}{2} \sum_{a b} \Lambda_{a b} Q_{a b}+N \ln \left(Z_{S}\right)\right)
\end{aligned}
$$

where

$$
Z_{S}=\operatorname{Tr}_{S^{a}} \exp \left(-\frac{1}{2} \sum_{a b} \Lambda_{a b} S^{a} S^{b}\right) .
$$

The same procedure is used for the Gaussian spins to yield

$$
\frac{\ln \left[\overline{Z^{n}}\right]}{N}=\operatorname{extr} S^{* *}[Q, P, \Lambda, \Gamma],
$$

where $S^{* *}[Q, P, \Lambda, \Gamma]$ is the saddle point action,

$$
\begin{aligned}
S^{* *}[Q, P, \Lambda, \Gamma]= & \frac{\beta^{2}}{2} \sum_{a b} Q_{a b} P_{a b}+\frac{1}{2} \sum_{a b} \Lambda_{a b} Q_{a b} \\
& +\frac{1}{2} \sum_{a b} \Gamma_{a b} P_{a b}+\ln \left(Z_{S}\right)+\ln \left(Z_{x}\right),
\end{aligned}
$$




$$
Z_{x}=\operatorname{Tr}_{x^{a}} \exp \left(-\frac{1}{2} \sum_{a b} \Gamma_{a b} x^{a} x^{b}\right)
$$

The saddle point equations with respect to $P_{a b}$ and $Q_{a b}$ allow the evaluation of the saddle values of the variables $\Lambda_{a b}$ and $\Gamma_{a b}$,

$$
\begin{aligned}
& \frac{\partial S^{* *}}{\partial P_{a b}}=0 \Rightarrow \Gamma_{a b}=-\beta^{2} Q_{a b}, \\
& \frac{\partial S^{* *}}{\partial Q_{a b}}=0 \Rightarrow \Lambda_{a b}=-\beta^{2} P_{a b},
\end{aligned}
$$

leading to the reduced saddle point $S^{*}[Q, P]$ such that

$$
\frac{\ln \left[\overline{Z^{n}}\right]}{N}=\operatorname{extr} S^{*}[Q, P]
$$

with

$$
\begin{aligned}
S^{*}[Q, P]= & -\frac{\beta^{2}}{2} \sum_{a b} Q_{a b} P_{a b} \\
& +\ln \left[\operatorname{Tr}_{S^{a}} \exp \left(\frac{\beta^{2}}{2} \sum_{a b} P_{a b} S^{a} S^{b}\right)\right] \\
& -\frac{1}{2} \operatorname{Tr} \ln (I-\beta Q)
\end{aligned}
$$

and where the Gaussian integral giving $Z_{x}$ has been evaluated. The saddle point value of $P_{a b}$ is given by

$$
\frac{\partial S^{*}}{\partial Q_{a b}} \Rightarrow P=\frac{1}{\beta}(I-\beta Q)^{-1}
$$

thus leading to the new reduced action $S[Q]$ such that

$$
\frac{\ln \left[\overline{Z^{n}}\right]}{N}=\operatorname{extr} S[Q]
$$

with

$$
\begin{aligned}
S[Q]= & \frac{1}{2}\left[n-\operatorname{Tr}(I-\beta Q)^{-1}-\operatorname{Tr} \ln (I-\beta Q)\right] \\
& +\ln \operatorname{Tr}_{S^{a}} \exp \left(\frac{\beta}{2} \sum_{a b}\left[(I-\beta Q)^{-1}\right]_{a b} S^{a} S^{b}\right) .
\end{aligned}
$$

The saddle point equation from Eq. (22) is

$$
-\frac{\beta}{2}(I-\beta Q)^{-2}+\frac{\beta}{2}(I-\beta Q)^{-1}+\frac{\beta^{2}}{2}(I-\beta Q)^{-2} A=0,
$$

where the elements of the matrix $A$ are given by

$$
A_{a b}=\frac{\operatorname{Tr}_{S^{a}} S^{a} S^{b} \exp \left(\frac{\beta}{2} \sum_{a b}\left[(I-\beta Q)^{-1}\right]_{a b} S^{a} S^{b}\right)}{\operatorname{Tr}_{S^{a}} \exp \left(\frac{\beta}{2} \sum_{a b}\left[(I-\beta Q)^{-1}\right]_{a b} S^{a} S^{b}\right)} .
$$

We use the fact that the matrix $(I-\beta Q)$ should not be singular at the saddle point (otherwise the saddle point lies on a branch cut) to conclude

$$
Q=A,
$$

which is the physical saddle point equation for $Q$. We proceed by studying the replica-symmetric form of this action with

$$
Q_{a b}^{R S}=(1-q) \delta_{a b}+q U_{a b},
$$

where $U_{a b}=1$ for all $(a, b)$. After some algebra one finds that

$$
\begin{aligned}
s(q)= & \lim _{n \rightarrow 0} \frac{S\left[Q^{R S}\right]}{n}=-\frac{1}{2} \ln [1-\beta(1-q)] \\
& -\frac{1}{2} \frac{\beta q[-1+2 \beta q(1-q)]}{[1-\beta(1-q)]^{2}}+\int \frac{d z}{\sqrt{2 \pi}} \\
& \times \exp \left(-\frac{z^{2}}{2}\right) \ln \left[2 \cosh \left(\frac{\beta z \sqrt{q}}{[1-\beta(1-q)]}\right)\right] .
\end{aligned}
$$

Taking the saddle point with respect to $q$ above yields

$$
\begin{aligned}
\frac{d s(q)}{d q}= & \frac{\beta^{2}}{2} \frac{(\beta q+\beta-1)}{[1-\beta(1-q)]^{3}}\left[\int \frac{d z}{\sqrt{2 \pi}} \exp \left(-\frac{z^{2}}{2}\right)\right. \\
& \left.\times \tanh ^{2}\left(\frac{\beta z \sqrt{q}}{[1-\beta(1-q)]}\right)-q\right] \\
= & 0 .
\end{aligned}
$$

There are two sets of possible solutions to Eq. (28):

$$
q=\frac{1}{\beta}-1
$$

and

$$
q=\int \frac{d z}{\sqrt{2 \pi}} \exp \left(-\frac{z^{2}}{2}\right) \tanh ^{2}\left(\frac{\beta z \sqrt{q}}{[1-\beta(1-q)]}\right)
$$

However, as mentioned above, the solution (29) is unphysical. The solution (30) corresponds to the physical solution (25) and only has the paramagnetic solution $q=0$ for $T$ $>T_{c}=2$. Hence for $T>T_{c}=2$ one is (in the replicasymmetric scheme) in a paramagnetic phase.

The energy per spin $E$ obtained from Eq. (22) is

$$
E=\lim _{n \rightarrow 0} \frac{1}{2 \beta}\left[1-\frac{1}{n} \operatorname{Tr}(1-\beta Q)^{-1}\right],
$$

where $Q_{a b}$ here is the solution to the saddle point equation. The replica-symmetric (RS) energy per spin is thus 


$$
E^{R S}=-\frac{1}{2}\left(\frac{(1-q)[1-\beta(1-q)]+q}{[1-\beta(1-q)]^{2}}\right) .
$$

Hence, for $T>T_{c}=2$, that is to say in the paramagnetic phase where one can be sure that the RS solution is correct, one has

$$
E=-\frac{1}{2(1-\beta)} .
$$

For $T \ll 1$ a low-temperature expansion of Eq. (30) yields

$$
q=1-\frac{T}{1+\sqrt{\pi / 2}}+O\left(T^{3}\right) .
$$

With the replica-symmetric ansatz, in the spin-glass phase, the ground-state energy is

$$
E_{G S}^{R S}=-\frac{1}{2}\left(\frac{2}{\pi}+\frac{2 \sqrt{2}}{\sqrt{\pi}}+1\right) .
$$

As in the SK model, if one computes the entropy corresponding to this replica-symmetric solution within the spin-glass phase, one finds a temperature where it becomes negative, indicating the need to break replica symmetry. We believe that the continuous nature of the transition, however, suggests that the underlying physics is the same as that of the SK model and the Landau expansion of $S\left[Q_{a b}\right]$ has the same generic form as that of the SK model, indicating a continuous replica symmetry breaking (RSB) which sets in at $T_{c}$. However, the RSB solution is very sensitive to the details and coefficients of the Landau expansion and this picture needs to be confirmed. The introduction of next-nearestneighbor interactions thus does not qualitatively change the behavior of the model, in agreement with the previously discussed intuitive notion that (for a given spin type) the behavior of the density of the largest eigenvalues determines the nature of the transition.

\section{NEGATIVE-TEMPERATURE $K<0$ MODEL}

The Hamiltonian (1) is not invariant under the transformation $K \rightarrow-K$. In this case the action for the replicated partition function is given by

$$
\begin{aligned}
S[Q]= & \frac{1}{2}\left[n-\operatorname{Tr}(I+\beta Q)^{-1}-\operatorname{Tr} \ln (I+\beta Q)\right] \\
& +\ln \operatorname{Tr}_{S^{a}} \exp \left(-\frac{\beta}{2} \sum_{a b}\left[(I+\beta Q)^{-1}\right]_{a b} S^{a} S^{b}\right) .
\end{aligned}
$$

In the high-temperature phase we expect the annealed approximation to be exact and find

$$
f_{\text {ann }}=-\frac{1}{\beta} \ln (2)+\frac{1}{2 \beta} \ln (1+\beta) .
$$

The annealed entropy per spin $S_{\text {ann }}$ is thus

$$
S_{\mathrm{ann}}=-\beta^{2} \frac{\partial f}{\partial \beta}=\ln (2)-\frac{1}{2} \ln (1+\beta)+\frac{\beta}{2(1+\beta)} .
$$

The annealed energy is given by

$$
E_{\mathrm{ann}}=\frac{1}{2(1+\beta)}
$$

The annealed solution can only be valid as long as $S_{\text {ann }}$ is positive. If there exists a $\beta_{\text {bound }}$ such that $S_{\text {ann }}\left(\beta_{\text {bound }}\right)<0$ for $\beta>\beta_{\text {bound }}$, then $\beta_{\text {bound }}$ gives us an upper bound for the inverse temperature at which the equilibrium transition occurs, $\beta_{K}$. In the Adam-Gibbs-Di Marzio scenario ${ }^{13} T_{K}=1 / \beta_{K}$ corresponds to the temperature where the configurational entropy $S_{\text {conf }}$ vanishes and the subindex $K$ in $\beta$ stands for the Kauzmann temperature which originates from the Kauzmann paradox ${ }^{14}$ which is based on the observation that an extrapolation of a high-temperature entropy cannot cross the lowtemperature solution. Sometimes $T_{K}$ is also referred to as the ideal glass transition temperature, not to be confused with the experimental glass transition temperature $T_{g}$ in the context of finite-dimensional glasses which turns out to be only a convention corresponding to an extremely large relaxation time as opposed to a diverging one. Because both entropies ( $S_{\text {ann }}$ and $S_{\text {conf }}$ ) are monotonically increasing functions of the temperature and $S_{\text {conf }}<S_{\text {ann }}$, this means that $\beta_{\text {bound }}>\beta_{K}$ is an upper bound of the ideal glass transition temperature $T_{K}$. One finds that $\beta_{\text {bound }} \approx 8.82$ and hence $T_{\text {bound }}=1 / \beta_{\text {bound }}$ $\approx 0.113$.

In the replica-symmetric ansatz the equation for the Edwards-Anderson order parameter $q$ is

$$
q=\int \frac{d z}{\sqrt{2 \pi}} \exp \left(-\frac{z^{2}}{2}\right) \tanh ^{2}\left(\frac{\beta z \sqrt{q}}{[1+\beta(1-q)]}\right) .
$$

The examination of this equation shows that one can no longer have a continuous phase transition from $q=0$. For low temperatures one can show that a solution with $q$ nonzero exists and that

$$
q=1-\frac{T}{\sqrt{\pi / 2}-1}+O\left(T^{2}\right)
$$

Numerically the inverse temperature $\beta_{c}^{R S}$ at which $q$ can become nonzero is found to be $\beta_{c}^{R S} \approx 29.3$, thus giving $T_{c}^{R S}$ $\approx 0.034$. This is much lower than $T_{K}$ above and hence the replica-symmetric solution cannot eliminate the entropy crisis at $T_{\text {bound }}$. Clearly one must resort to a replica-symmetrybroken ansatz. Guided by the results of our RS calculation and the numerical simulations we make the random energy model (REM) (one-step) like ansatz where the matrix $Q$ is given by $n / m$ matrices $\widetilde{Q}$ about the diagonal of $Q$ of size $m \times m$ and is zero outside these blocks. The matrix $\widetilde{Q}$ takes the form

$$
\widetilde{Q}=I(1-q)+q \widetilde{U},
$$


where $I$ is the identity matrix and $\widetilde{U}$ is the matrix with each element equal to 1 . We note the following results:

$$
\begin{aligned}
(I+\beta \widetilde{Q})^{-1}= & \frac{1}{1+\beta(1-q)} I \\
& +\frac{\beta q \widetilde{U}}{[1+\beta(1-q)][1+\beta(1-q)+\beta m q]},
\end{aligned}
$$

$$
\begin{aligned}
\operatorname{Tr}(1+\beta Q)^{-1} & =\frac{n}{m} \operatorname{Tr}(1+\beta \widetilde{Q})^{-1} \\
& =\frac{n}{m}\left(\frac{1}{1+\beta(1-q)+\beta m q}+\frac{m-1}{1+\beta(1-q)}\right),
\end{aligned}
$$

$$
\begin{aligned}
\operatorname{Tr} \ln \left[(1+\beta Q)^{-1}\right]= & \frac{n}{m} \operatorname{Tr} \ln \left[(1+\beta \widetilde{Q})^{-1}\right] \\
= & \frac{n}{m}\{\ln [1+\beta(1-q)+\beta q m] \\
& +(m-1) \ln [1+\beta(1-q)]\},
\end{aligned}
$$

and

$$
\begin{aligned}
\ln \operatorname{Tr}_{S^{a}} \exp \left(\frac{\beta}{2} \sum_{a b}\left[(I-\beta Q)^{-1}\right]_{a b} S^{a} S^{b}\right) \\
=\frac{n}{m} \ln \operatorname{Tr}_{S^{a}} \exp \left(\frac{\beta}{2} \sum_{a b}\left[(I+\beta \widetilde{Q})^{-1}\right]_{a b} S^{a} S^{b}\right) \\
=\frac{n \beta}{2[1+\beta(1-q)]}+\frac{n}{m} \ln \left[\int \frac{d z}{\sqrt{2 \pi}} \exp \left(-\frac{1}{2} z^{2}\right)\right. \\
\left.\quad \times\{2 \cosh [\beta z \alpha(q, m, \beta)]\}^{m}\right],
\end{aligned}
$$

where

$$
\alpha(q, m, \beta)=\frac{\sqrt{q}}{\sqrt{[1+\beta(1-q)][1+\beta(1-q)+\beta m q]}} .
$$

One therefore finds the one-step action

$$
\begin{aligned}
s(q, m)= & \lim _{n \rightarrow 0} \frac{S\left[Q^{1 R S B}\right]}{n} \\
= & \frac{1}{2}\left[\frac{-\beta^{2} q(1-q+m q)}{[1+\beta(1-q)][1+\beta(1-q)+m \beta q]}\right. \\
& -\frac{1}{m} \ln [1+\beta(1-q)+m \beta q] \\
& \left.-\left(1-\frac{1}{m}\right) \ln [1+\beta(1-q)]\right]+\frac{1}{m} \ln [z(q, m)],
\end{aligned}
$$

where

$$
z(q, m)=\int \frac{d z}{\sqrt{2 \pi}} \exp \left(-\frac{1}{2} z^{2}\right)\{2 \cosh [\beta z \alpha(q, m, \beta)]\}^{m}
$$

One finds that

$$
\begin{aligned}
& \frac{\partial}{\partial q} s(q, m) \\
& =\beta^{2} \alpha(q, m, \beta) \frac{\partial \alpha(q, m, \beta)}{\partial q}(1-m) \\
& \quad \times\left[q-\frac{1}{z(q, m)} \int \frac{d z}{\sqrt{2 \pi}} \exp \left(-\frac{1}{2} z^{2}\right)\right. \\
& \left.\quad \times\{2 \cosh [\beta z \alpha(q, m, \beta)]\}^{m} \tanh ^{2}[\beta \alpha(q, m, \beta) z]\right]
\end{aligned}
$$

The saddle point of $s(q, m)$ corresponding to the solution (25) is thus

$$
\begin{aligned}
q= & \frac{1}{z(q, m)} \int \frac{d z}{\sqrt{2 \pi}} \exp \left(-\frac{1}{2} z^{2}\right)\{2 \cosh [\beta z \alpha(q, m, \beta)]\}^{m} \\
& \times \tanh ^{2}[\beta \alpha(q, m, \beta) z] .
\end{aligned}
$$

If one considers the case $m=1$, this solution must give the same free energy as the replica-symmetric free energy, which above the static transition temperature is the annealed free energy. However, one can find a nonzero value of $q$ which signals a dynamical transition where the system becomes stuck in metastable states of high free energy. Setting $m=1$ in Eq. (51) gives

$$
\begin{aligned}
q= & \exp \left(-\frac{1}{2} \beta^{2} \alpha(q, 1, \beta)^{2}\right) \int \frac{d z}{\sqrt{2 \pi}} \exp \left(-\frac{1}{2} z^{2}\right) \\
& \times \cosh [\beta z \alpha(q, 1, \beta)] \tanh ^{2}[\beta \alpha(q, 1, \beta) z]
\end{aligned}
$$

For small $\beta$ this equation has only the solution $q=0$. However, at $\beta_{d} \approx 7.325\left(T_{d}=1 / \beta_{d} \approx 0.137\right)$ one finds a nonzero value of $q$ with $q \approx 0.922$. This transition corresponds to what is known as the mode-coupling transition in modecoupling theories of the glass transition in their idealized version. $^{15}$

The precise way to locate both transitions ( $T_{d}$ and $T_{K}$ ) was suggested in a series of papers by Kirkpatrick et al. ${ }^{16}$ and later on applied to several models such as the random orthogonal model, ${ }^{17}$ Potts glasses, ${ }^{18}$ and mean-field quantum spin glasses. ${ }^{19}$ The static transition is located by expanding the corresponding free energy $f(q, m)$ about $m=1$ and writing

$$
\beta f(q, m)=\beta f_{\text {para }}+(m-1) V(q)+O\left((m-1)^{2}\right),
$$

where $f_{\text {para }}=f(0,1)$ is the paramagnetic free energy and 


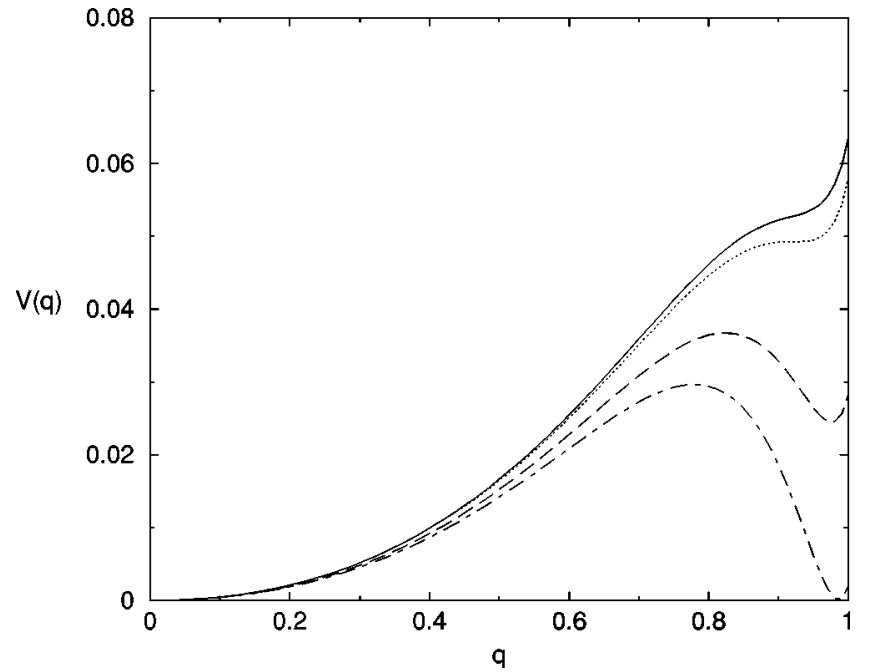

FIG. 1. Behavior of the potential $V(q)$ on lowering $T$. The different regimes are $T>T_{d}$ (solid line), $T=T_{d}$ (dotted line), $T_{K}<T$ $<T_{d}$ (dashed line), and $T=T_{K}$ (dot-dashed line).

$$
V(q)=\left.\beta \frac{\partial f(q, m)}{\partial m}\right|_{m=1}=-\left.\frac{\partial s(q, m)}{\partial m}\right|_{m=1}
$$

The static transition $T_{K}$ is given by the values of $\beta_{K}$ where $V\left(q_{K}\right)=V^{\prime}\left(q_{K}\right)=0$. The dynamic transition $T_{d}$ is given by the conditions $V^{\prime}\left(q_{d}\right)=V^{\prime \prime}\left(q_{d}\right)=0$ which correspond to the marginality condition and coincide with the solution found at $m=1$ and reported above.

The explicit form of the potential $V$ is

$$
\begin{aligned}
V(q)= & \frac{\beta q(1+2 \beta)}{2(1+\beta)[1+b(1-q)]}+\frac{1}{2} \ln [1+\beta(1-q)] \\
& -\frac{1}{2} \ln (1+\beta)-\exp \left(-\frac{1}{2} \beta^{2} \alpha(q, m, \beta)^{2}\right) \\
& \times \int \frac{d z}{\sqrt{2 \pi}} \exp \left(-\frac{1}{2} z^{2}\right) \\
& \times \cosh [\beta z \alpha(q, 1, \beta)] \ln \{\cosh [\beta \alpha(q, 1, \beta) z]\} .
\end{aligned}
$$

Using this to compute the static transition one finds that this gives $T_{K} \approx 0.116$ and $q_{K} \approx 0.985$. The behavior of $V(q)$ on lowering $T$ is shown in Fig. 1.

Note that at $T_{d}$ the value of the potential $V\left(q_{d}\right)$ is finite and corresponds to the configurational entropy at that temperature. As $T$ decreases below $T_{d}$, the analytical continuation of the solution $q_{d}$ to the saddle point of Eq. (48) increases while the value of $m_{d}(T)$ decreases. The configurational entropy evaluated at $T<T_{d}$ is $S_{c}(T)$ $=\beta\left[f\left(q_{d}(T), m_{d}(T)\right)-f_{\text {para }}(T)\right]$ and decreases while $T$ decreases down to a temperature $T_{K}$ where it vanishes and $q_{K}$ is the analytically continued value of $q_{d}\left(T_{K}\right)$.
Hence we see that in the case $K<0$ the physics of the problem is drastically altered by the interaction matrix and that the model now exhibits the phenomenology of a (meanfield) structural glass.

\section{NUMERICAL SIMULATIONS}

We have verified the main predictions for the SIM-SK model with positive and negative temperature by doing some numerical simulations in both cases. For the positivetemperature model we checked that the transition indeed occurs at $T=2$. This has been done applying standard finitesize scaling techniques useful for investigating small-size systems. For the negative model we found that strong freezing occurs at the mode-coupling temperature $T_{d}=0.137$ as happens for other models such as the $p$-spin ${ }^{20}$ or the random orthogonal model (Ref. 17).

\section{A. Some details of the simulations}

Simulations consist of standard Monte Carlo annealings using the Metropolis algorithm. The system is cooled down from high temperatures (typically twice the value of $T_{c}$ for the $K>0$ model and twice $T_{d}$ for the $K<0$ case). Annealing schedules are as follows: every $\Delta T=0.2$ for the $K>0$ model and every $\Delta T=0.01$ for the $K<0$ model the system is allowed to equilibrate over 1000 Monte Carlo steps (MCS) and statistics are collected during $10^{5} \mathrm{MCS}$ at each MCS. The sizes are small, $N=25,50,75,100$, but enough to locate the transition with some precision. The simulated range of temperatures is from $T=4$ down to $T=0.2$ for the $K>0$ model and from $T=0.3$ down to $T=0.01$ for the $K<0$ model. The number of samples were several thousands for all sizes.

Due to the long-range character of the interactions, the dependence of the time needed to do a MCS grows quite fast with the size of the system (actually like $N^{2}$ ). Therefore, for the statics, we had to limit our investigation to relatively small sizes. Moreover, a careful study of the relevant parameters for the transition (such as the kurtosis or the $G$ parameter to be defined below) requires a large number of samples (this is especially true for parameters like $G$ which measure sample-to-sample fluctuations). This last parameter is the most successful example of what are referred to as orderparameter fluctuation parameters (OPF parameters). ${ }^{21}$

Before showing the results let us mention that, while for the positive- $K$ model we achieved thermalization in a range of temperatures in the vicinity of $T_{c}$, for the negative- $K$ model thermalization was hardly achieved due to the quite small acceptance rate for all the temperatures simulated. This behavior is due to the small value of the relevant temperatures of the $K<0$ model where the transition occurs (one order of magnitude smaller). Because the typical energy change for both models is the same, the Boltzmann factor is drastically reduced for changes which increase the energy in the $K<0$ model as compared to the $K>0$ case. This implies a very small acceptance rate for the $K<0$ models as compared to the $K>0$ case. In Fig. 2 we show the acceptance as a function of $T$ for the two cases. Note that the acceptance is nearly two orders of magnitude smaller in the negative 


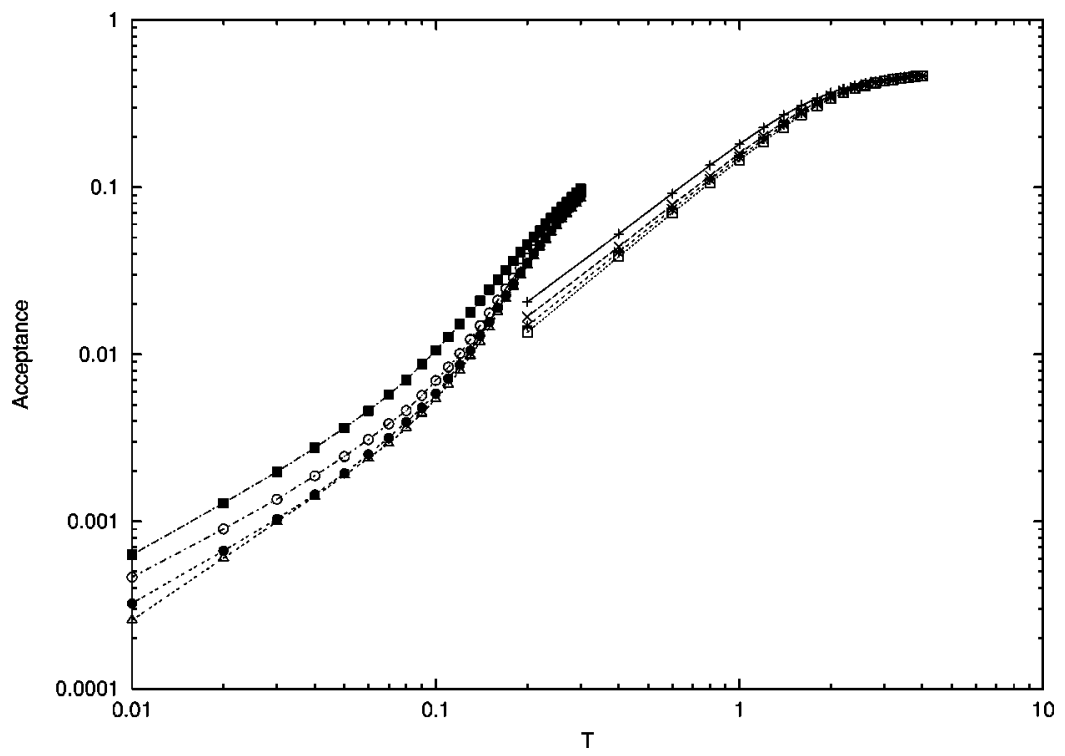

FIG. 2. Acceptance rate as a function of $T$ in the relevant temperature ranges for the $K>0$ (right set of curves) and $K<0$ (left set of curves) models. For each set and from top to bottom we have $N=25,50,75,100$. Note that the acceptance rate is typically 10 times smaller in the $K<0$ model. model as compared to the positive model. Hence a good sampling of the configurational space for the negative model can be excluded.

\section{B. Positive-temperature $K>0$ model}

In this model there is a continuous RSB transition at $T_{c}$ $=2$. The energy and the specific heat are shown as a function of $T$ in Figs. 3 and 4. We also plot the result for the annealed expression only valid above the critical temperature. As we see the behavior of these quantities is similar to what is found for the SK model: the maximum of the specific heat occurs below $T_{c}$.

The transition can be well determined by looking at the $B$ and $G$ parameters as functions of the temperature. These parameters are defined by

$$
B=\frac{1}{2}\left(3-\frac{\overline{\left\langle q^{4}\right\rangle}}{\left(\overline{\left\langle q^{2}\right\rangle}\right)^{2}}\right),
$$

$$
G=\frac{\overline{\left(\left\langle q^{2}\right\rangle\right)^{2}}-\left(\overline{\left\langle q^{2}\right\rangle}\right)^{2}}{\overline{\left\langle q^{4}\right\rangle}-\left(\overline{\left\langle q^{2}\right\rangle}\right)^{2}}
$$

In the infinite-size limit these parameters behave as $B(T)=\hat{B}(T) \theta_{H}\left(T_{c}-T\right)$ while the behavior of $G$ turns out to be simpler, $G(T)=\frac{1}{3} \theta_{H}\left(T_{c}-T\right)$, and hence transpires to be a better indicator for the transition. In Fig. 5 we show the kurtosis parameter $B$ as a function of $T$ for different sizes. In the same way as in the SK model ${ }^{21}$ we find a crossing at a temperature close to $T_{c}=2$. This crossing turns out to be also present for the OPF parameter $G$ as shown in Fig. 6.

\section{Negative-temperature $K<0$ model}

As mentioned previously, we were not able to thermalize for this case close to the transition, so we do not have good data for order parameters such as $B$ or $G$. We show the results for the energy and specific heat in Figs. 7 and 8.

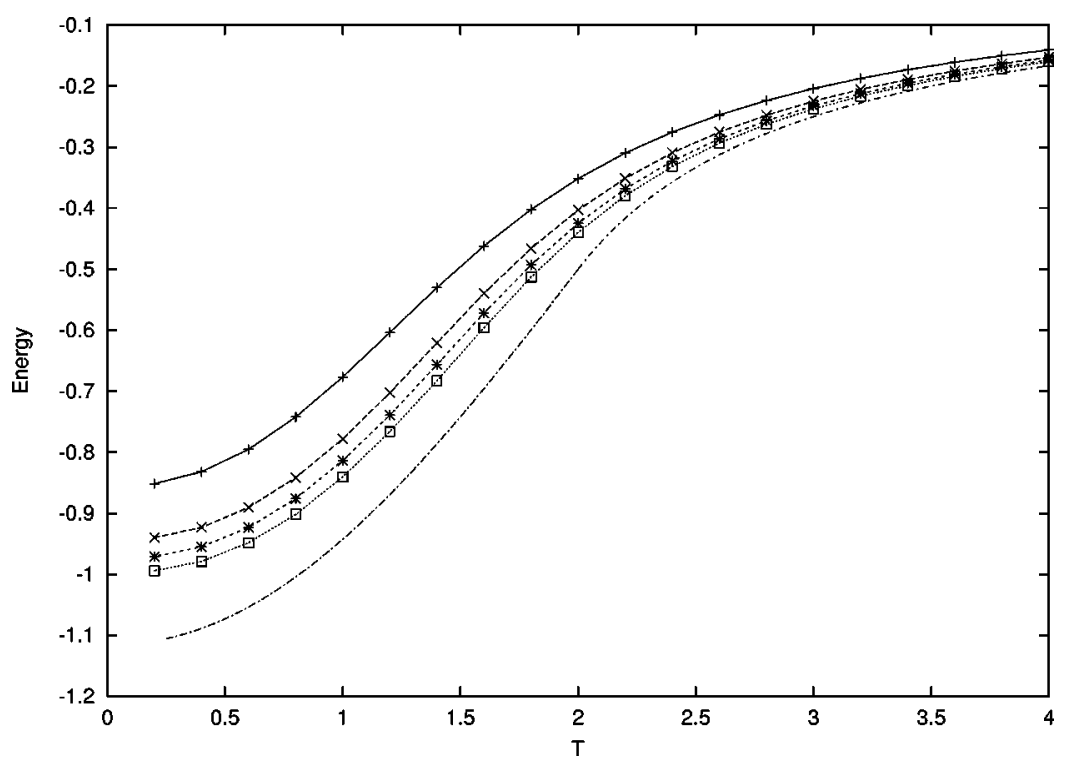

FIG. 3. Energy as a function of $T$ for the model $K>0$. From top to bottom we have $N$ $=25,50,75,100$. The solid line is the replicasymmetric result. 


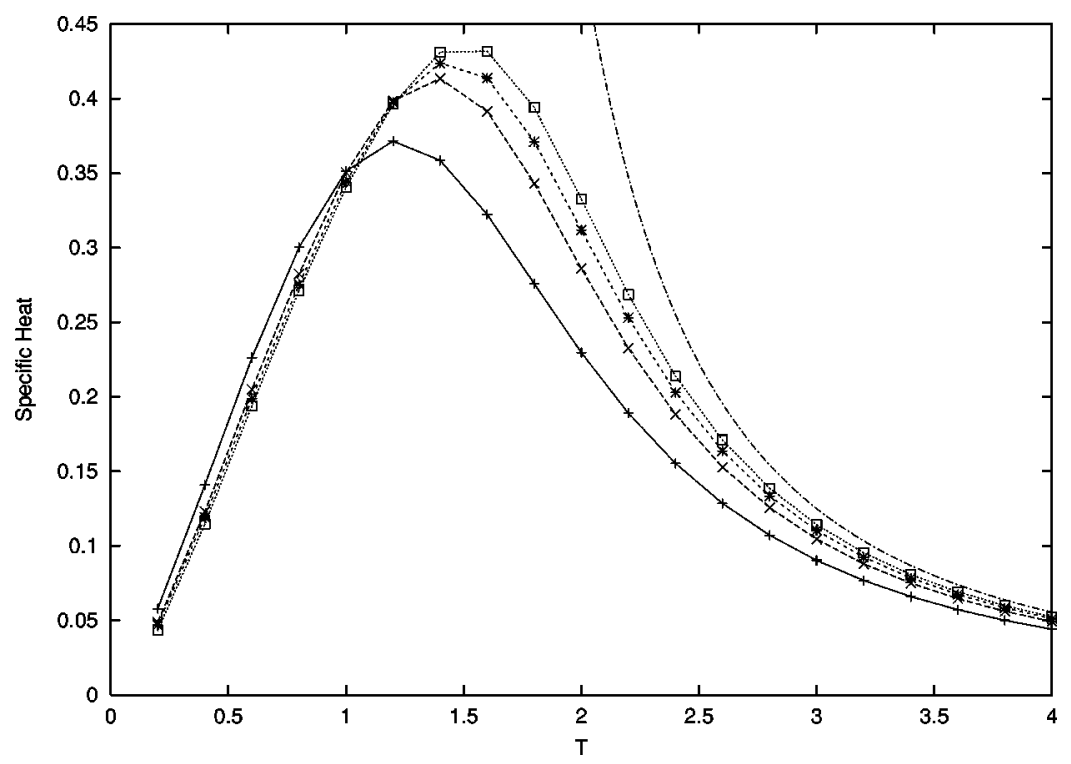

FIG. 4. Specific heat as a function of $T$ for the model $K>0$. From bottom to top at high $T$ we have $N=25,50,75,100$. The solid line is the high$T$ result.

As we said before we did not succeed in thermalizing the model at low temperatures to extract the behavior of the order parameters. Consequently we do not have good data for the kurtosis $B$, Eq. (56), and the OPF $G$, Eq. (57). The only transition about which we may have some hints is the dynamical transition where the dynamics becomes extremely slow. One could be tempted to interpret the crossing point for the specific heat at different sizes (Fig. 8) as the signature of that dynamical transition. Despite the fact that this interpretation seems reasonable, we are not absolutely certain and so we prefer to leave this question open.

More evidence for the dynamical transition can be obtained by doing annealing experiments for finite cooling rates and very large sizes. One of these cooling experiments, shown in Fig. 9 for a system of size $N=1000$, was cooled down to very low temperatures staying for $10^{5}$ MCS at each temperature and changing $T$ by 0.01 . The system gets trapped below a crossover temperature $T^{*} \sim 0.14$ and starts to deviate from the high- $T$ line. We plot two independent dynamical histories to demonstrate how the departure from the ergodic line depends on the dynamical history signaling the presence of nonergodic effects in the dynamics (below $T^{*}$ the typical relaxation time has become much larger than the time the system remains at each temperature, i.e., $10^{5}$ ).

Note that in this dynamical study the static transition $T_{K}$ remains completely hidden. At this temperature no divergence of a relaxation time can be observed since that time has already diverged above at the dynamical transition. The only way to show the existence of this transition is by estimating the configurational entropy and determining the temperature at which that quantity vanishes. Such a task has been successfully undertaken in the case of the randomorthogonal model $^{22}$ which displays a very similar behavior to the $K<0$ model and requires the use of projection techniques, in the spirit of those introduced by Stillinger and

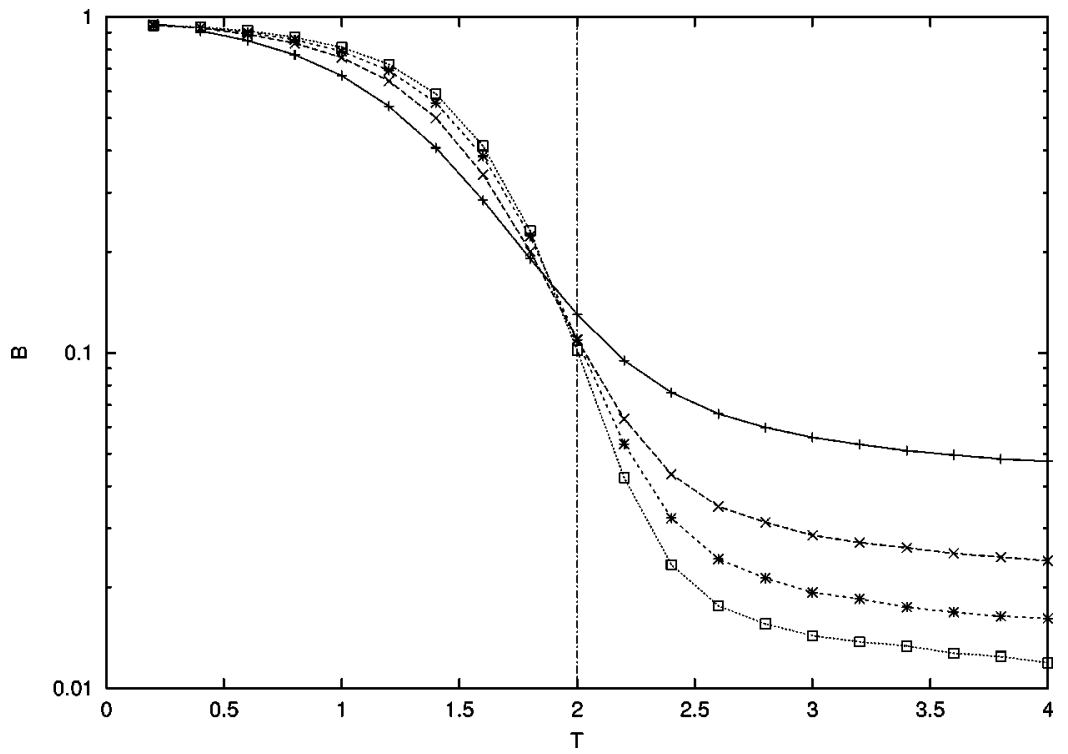

FIG. 5. Kurtosis $B$ as a function of $T$ for the model $K>0$. From top to bottom we have $N$ $=25,50,75,100$. The crossing temperature shifts with the size towards $T_{c}=2$. 


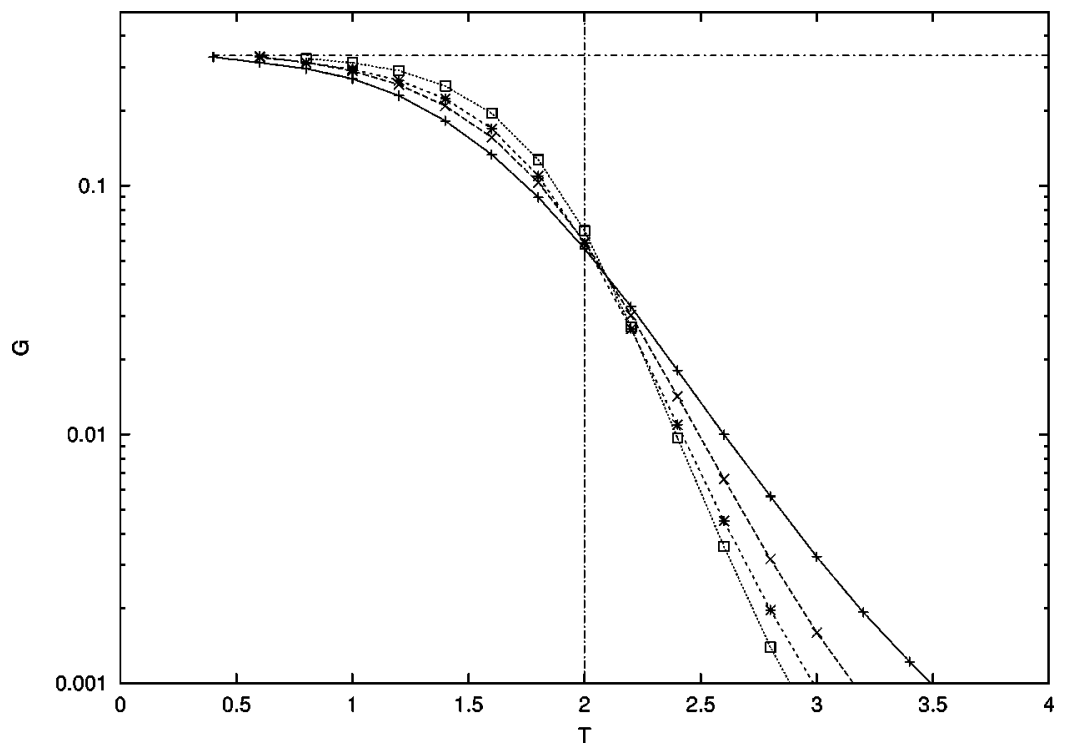

FIG. 6. OPF $G$ as a function of $T$ for the model $K>0$. From top to bottom at low $T$ we have $N=25,50,75,100$. The different curves cross close to $T_{c}=2$ where $G_{c} \simeq 0.057$.

Weber, for the study of the potential energy landscape in the context of glasses. ${ }^{23}$

\section{CONCLUSIONS}

In this paper we introduced a solvable model of a spin glass corresponding to a mean-field version of a spin-glass model with particular nearest- and next-nearest-neighbor interactions. The main interest of the model is that it shows how correlations in the couplings may completely change the character of the spin-glass transition from continuous replica symmetry breaking to a one-step transition. We analyzed two cases: the positive model $K>0$ with a phase transition and behavior similar to that of the SK model and the $K<0$ model with ideal mode-coupling behavior and a phase transition similar to the ROM or to the $p$-spin model. The different character of the transition in both models can be ascribed to the different behavior of the eigenvalue density distribution close to the maximum eigenvalue or threshold. For the $K$ $>0$ the distribution vanishes at the threshold (i.e., $\lambda_{+}=4$ ) and the equilibrium distribution condensates around configurations in the vicinity of the maximum eigenvector. For the $K<0$ the eigenvalue density diverges at the threshold (i.e., $\left.\lambda_{-}=0\right)$. In that case, the equilibrium configuration condensates around an extremely large number of eigenvectors with eigenvalue close to 0 . Hence, the phase space splits into a very large number (exponentially large with the size of the system) of ergodic components or phases, these phases corresponding to different eigenvectors with eigenvalue very close to the threshold which extensively contribute to the configurational entropy. Obviously, eigenvectors of the coupling matrix never coincide with possible configurations of the Ising system, so this argumentation must be taken only as a rough picture. Nevertheless, the idea that the type of eigenvalue distribution determines the character of the transition seems quite intuitive. Actually, if one considers spherical instead of Ising spins, then the transition disappears for the $K$ $<0$ model but persists for the $K>0$ case. For the $K<0$ model the transition disappears because there is no longer a vanishing of the configurational entropy (the classical nature

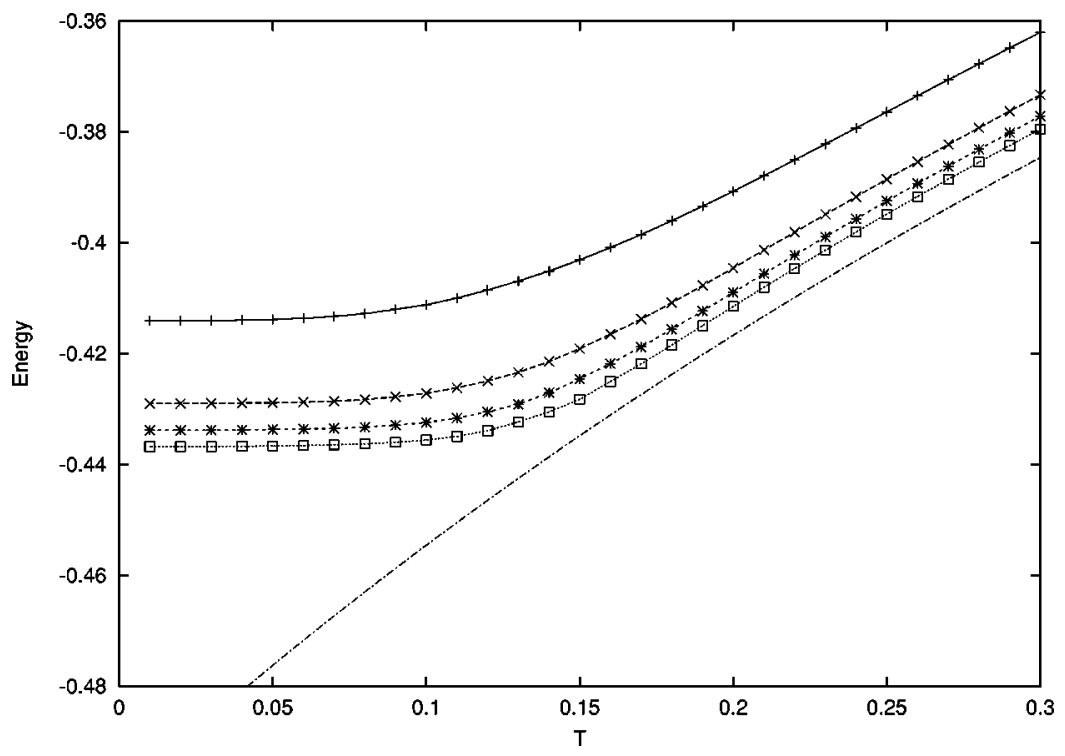

FIG. 7. Energy as a function of $T$ for the model $K<0$. From top to bottom we have $N=25,50,75,100$. The solid line is the high- $T$ result. 


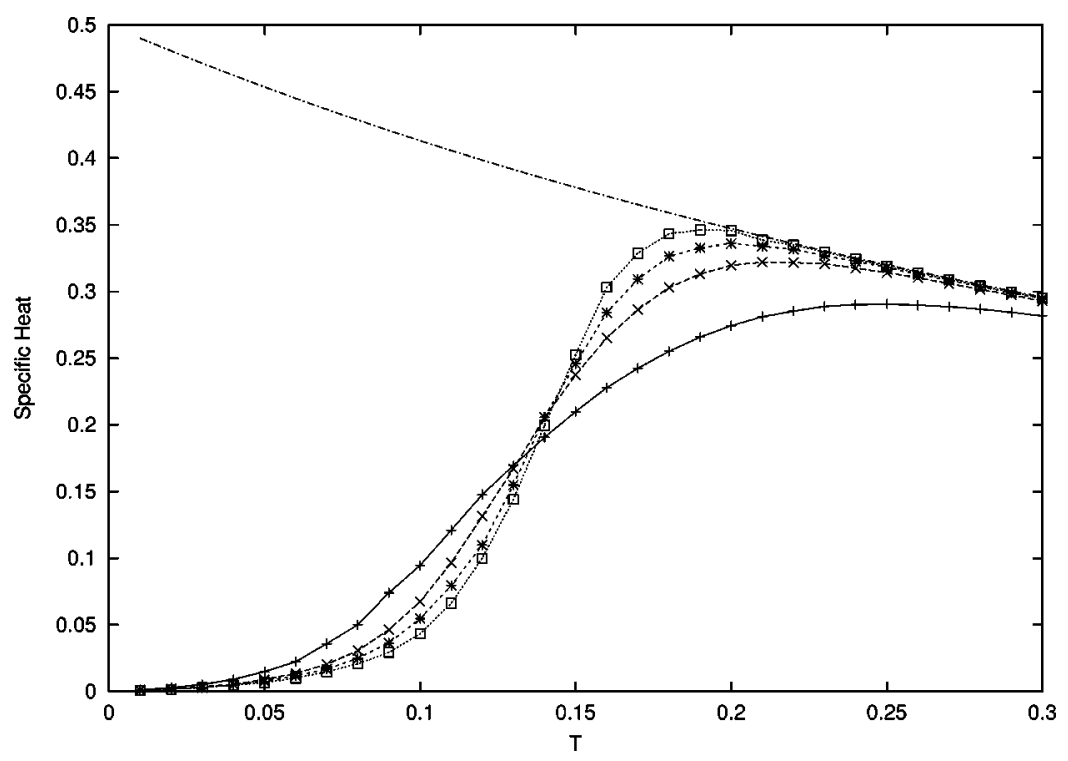

FIG. 8. Specific heat as function of $T$ for the model $K<0$. From top to bottom at low $T$ we have $N=25,50,75,100$. The solid line is the high$T$ result.

of spins allows for a negative entropy). The same mechanism occurs in the ROM where the one-step transition with Ising spins disappears in case of spherical spins. In this case, the density of eigenvalues is given by $\rho(\lambda)=\frac{1}{2} \delta(\lambda-1)+\frac{1}{2} \delta(\lambda$ $+1)$, showing that the density diverges in the vicinity of the maximum eigenvalue.

What is the real connection between the density of eigenvalues and the order of the transition? The mechanism which makes a continuous transition become first order (in the order parameter) in spin-glass theory is well known and based on the role of the so-called complexity or (sometimes misleadingly) configurational entropy. ${ }^{16,24,25}$ In mean-field theory the complexity $S_{c}(F, T)$ at a given free energy and temperature corresponds to the logarithm of the number of Thouless-Anderson-Palmer (TAP) free energy minima solutions with that free energy and temperature. The complexity defines the so-called potential $\Phi(F, T)=F-T S_{c}(F, T)$. This quantity displays two types of different behaviors at low enough temperatures. For models with continuous replica symmetry breaking $\Phi$ has a single minimum at the equilib- rium free energy value $F_{\text {eq }}(T)$ while for models with a onestep scenario this function may display a minimum at a threshold value $F^{*}(T)$ higher that the equilibrium free energy $F_{\text {eq }}(T)$. The difference $\beta\left[F^{*}(T)-F_{\text {eq }}(T)\right]$ defines the complexity at that temperature $S^{*}(T)=S_{c}\left(F^{*}(T), T\right)$ which is positive for one-step models but vanishes in continuous models. Because at high free energies $\Phi(F, T) \sim F$ it is clear that the abundance of metastable solutions makes $S_{c}$ always larger, raising the possibility that the potential $\Phi(F, T)$ displays a minimum above the equilibrium free energy. Therefore, a divergence of the eigenvalue density at the lowest value goes in the appropriate direction of generating a minimum in $\Phi$ and hence making the transition become first order in the order parameter.

As for further directions of interest we mention the investigation of the existence of a model interpolating between the $K>0$ and $K<0$ models. This would be very interesting to understand better the mechanism which makes the spin-glass transition change from a continuous one to a discontinuous one, as well as to connect this change with the behavior of

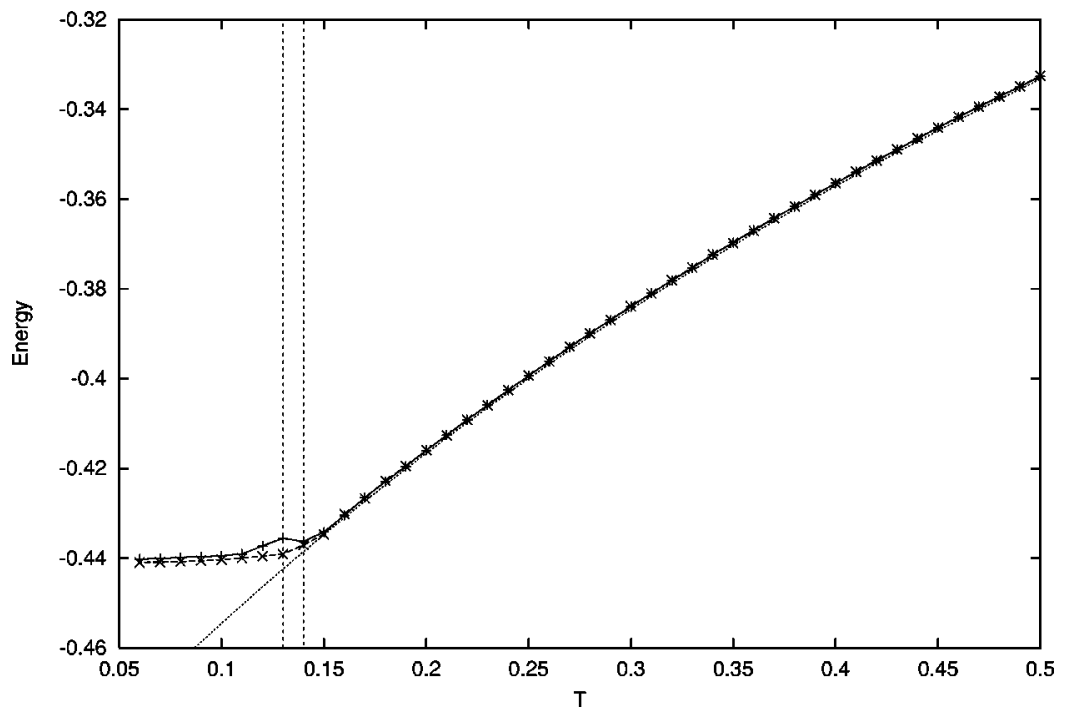

FIG. 9. Cooling experiments in the model $K$ $<0$. The two vertical dashed lines limit the range of temperatures $0.13-0.14$. The dotted line is the high- $T$ result. 
the density of eigenvalues close to the threshold. In this direction it would be also interesting to investigate in a general way the type of phase transition for a generic eigenvalue distribution using analytical techniques such as those developed for the ROM. ${ }^{17}$

It would also be interesting to investigate the behavior of the different terms in the TAP expansion to see how this change of behavior occurs and how this is related to the geometrical properties of the free energy landscape. Note that the information contained in the density of eigenvalues is closely related to the topological features of the energy landscape. Therefore, it is reasonable to think that the nature of the transition in these models arises from the geometrical properties of the energy (and hence, the free energy) landscape.

Finally, we would like to investigate the behavior of this model on a finite-dimensional lattice. In the simplest way this can be done taking $J$ to be nearest-neighbor Gaussian matrix and defining $K$ according to Eq. (2). It would be very interesting to see how the two universality classes describing the mean-field behavior are modified in the presence of activated processes and how they manifest themselves in the dynamical properties of realistic systems. This could give additional understanding about why some models behave in one way or another and how topological properties of the landscape influence the dynamical behavior of real systems.

\section{ACKNOWLEDGMENTS}

We are grateful to A. Lefèvre and M. Sales for a careful reading of the manuscript. F.R. is supported by the Ministerio de Ciencia y Tecnología in Spain, Project No. BFM20013525, and Generalitat de Catalunya.
*Electronic address: dean@irsamc.ups-tlse.fr

†Electronic address: ritort@ffn.ub.es

${ }^{1}$ D. Sherrington and S. Kirkpatrick, Phys. Rev. Lett. 35, 1792 (1975); Phys. Rev. B 17, 4348 (1978).

${ }^{2}$ K. Binder and A.P. Young, Rev. Mod. Phys. 58, 801 (1986); M. Mézard, G. Parisi, and M. A. Virasoro, Spin Glass Theory and Beyond (World Scientific, Singapore, 1987); K. H. Fischer and J. A. Hertz, Spin Glasses (Cambridge University Press, Cambridge, England, 1991); V. Dotsenko, Introduction to the Replica Theory of Disordered Statistical Systems (Cambridge University Press, Cambridge, England, 2000).

${ }^{3}$ S.F. Edwards and P.W. Anderson, J. Phys. F: Met. Phys. 5, 965 (1975).

${ }^{4}$ G. Parisi, Phys. Rev. Lett. 43, 1754 (1979); J. Phys. A 13, 1101 (1980).

${ }^{5}$ D.S. Fisher and D.A. Huse, Phys. Rev. Lett. 56, 1601 (1986); A.J. Bray and M.A. Moore, ibid. 58, 57 (1987).

${ }^{6}$ M. L. Mehta Random Matrices and the Statistical Theory of Energy Levels (Academic, New York, 1967).

${ }^{7}$ J.M. Kosterlitz, D.J. Thouless, and R.C. Jones Phys. Rev. Lett. 36, 1217 (1976).

${ }^{8}$ W.A. Little, Math. Biosci. 19, 101 (1974).

${ }^{9}$ In the nonsymmetric case the density of eigenvalues can be calculated within the replica framework.
${ }^{10}$ J.J. Hopfield, Proc. Natl. Acad. Sci. U.S.A. 79, 2554 (1982).

${ }^{11}$ S. Cabasino, E. Marinari, P. Paolucci, and G. Parisi, J. Phys. A 21, 4201 (1988).

${ }^{12}$ R. Brunetti, G. Parisi, and F. Ritort, Phys. Rev. B 46, 5339 (1992).

${ }^{13}$ J.H. Gibbs and E.A. Di Marzio, J. Chem. Phys. 28, 373 (1958); G. Adams and J.H. Gibbs, ibid. 43, 139 (1965).

${ }^{14}$ W. Kauzmann, Chem. Rev. 43, 219 (1948).

${ }^{15}$ E. Leutheusser, Phys. Rev. A 29, 2765 (1984); T.R. Kirkpatrick, ibid. 31, 939 (1985); W. Götze and L. Sjogren, Rep. Prog. Phys. 55, 241 (1992).

${ }^{16}$ T.R. Kirkpatrick and P.G. Wolynes, Phys. Rev. B 36, 8552 (1987); T.R. Kirkpatrick, D. Thirumalai, and P.G. Wolynes, Phys. Rev. A 40, 1045 (1989).

${ }^{17}$ E. Marinari, G. Parisi, and F. Ritort, J. Phys. A 27, 7647 (1994).

${ }^{18}$ E. De Santis, G. Parisi, and F. Ritort, J. Phys. A 28, 3025 (1995).

${ }^{19}$ F. Ritort, Phys. Rev. B 55, 14096 (1997).

${ }^{20}$ A. Crisanti, H. Horner, and H.J. Sommers, Z. Phys. B: Condens. Matter 92, 257 (1993).

${ }^{21}$ M. Picco, F. Ritort, and M. Sales, Eur. Phys. J. B 19, 565 (2001).

${ }^{22}$ A. Crisanti and F. Ritort, J. Phys.: Condens. Matter 14, 1381 (2002).

${ }^{23}$ F.H. Stillinger and T.A. Weber, Phys. Rev. A 25, 978 (1982).

${ }^{24}$ R. Monasson, Phys. Rev. Lett. 75, 2847 (1995).

${ }^{25}$ M. Mezard, Physica A 265, 352 (1999). 